\title{
Broadband Space tapered Golden Ratio Spiral Phased Arrays
}

\author{
A. El-makadema and A.K. Brown
}

The University of Manchester

\begin{abstract}
The paper discusses the effect of different space tapering techniques on the broadband performance of a class of sparse phased arrays based on the golden ratio configuration (Boeringer 2002). Different space tapering techniques are applied using a finite number of elements enclosed within a defined aperture size in order to demonstrate the effect of the geometry on the broadband performance. The paper shows how the element locations can be manipulated to achieve high gain and low side lobes. Tradeoffs are presented between side lobe level and gain for various space tapers over multi-octave bandwidth.
\end{abstract}

\section{Introduction}

The design of antenna arrays varies significantly depending on the desired performance requirements. In this paper the main concern is the required directivity, bandwidth and side lobe performance. The aim is to be able to meet these requirements with the least over all complexity that is the total number of elements needed and their relative positions in the array.

In any antenna array system with $N$ number of isotropic elements, the maximum possible gain that can be achieved occurs at a wavelength related to the relative positions of the antenna elements. In linear periodic arrays the maximum possible gain is achieved when the inter-element separation is approximately $0.9 \lambda^{\mathrm{a}}$. Since the array gain is related to the total radiation pattern from all the elements and not the linear summation of individual antenna gains, it is possible to arrange the antenna elements in such away that a higher array gain can be achieved than $N x G$ over a narrow band, where $N$ is the total number of elements in the array and $G$ is the gain of one element. The $N x G$ figure can however be used as a useful quality measure of the array gain over a broadband.

The maximum possible gain over the frequency band from $N$ available antenna elements occurs at a narrow bandwidth centred on $f_{\text {max }}$. For frequencies less than $f_{\text {max }}$ the gain of the array will reduce becoming less than $N x G$ at much lower frequencies. This is due to antenna element separations becoming closer together in terms of wavelength, which reduces or eliminates their contribution to the total array gain. The array now is oversampled and contains many redundant elements. On the other hand, for frequencies above $f_{\text {max }}$ the gain will also drop, as the element separation becomes far apart in terms of wavelength ultimately causing high peaks at angles other than the main beam, and thus reducing the total gain. This general trend is true irrespective of the array configuration

In order to design a cost efficient broadband array $f_{\text {max }}$ should be equal to $f_{\text {Low }}$. This is in order to avoid any elements becoming redundant at the low frequencies. This means that the array is under sampled at higher frequencies and therefore, the element positions must be optimised in order to minimize the effect of under sampling on the side lobes and the loss of gain.

\footnotetext{
a This is true provided the array is of the order of 8 elements or more (Howard 1981)
}

This optimization however, comes at the expense of reducing $G_{\max }$. In other words, a cost efficient broadband antenna array trades off maximum possible gain at a narrow band, with gain and side lobe performance over the total bandwidth.

In some broadband antenna array applications higher gain is required at a certain portion of the bandwidth where for example high noise sources may exist. In other applications a stable gain over the bandwidth is more of interest. Therefore, i is useful to be able to control the gain performance over the bandwidth in order to meet the system requirements with the minimum number of elements.

In order to see the effect on array performance from a pure geometrical point of view, a method is presented whereby different array geometries are compared using fixed number of elements enclosed within a constant circular aperture. The an tenna element assumed is a hemispherical element with a gain of $3 \mathrm{dBi}$ and the performance of the array assumed is now governed purely by the relative positions of antenna elements "that is the mutual coupling is ignored".

The Golden Ratio Spiral has been reported before for phased arrays (Boeringer 2002; Vigan 2000) for the purpose of narrowband and multiple beam applications. In this paper the Golden ratio spiral is found to possess attractive properties that are useful for designing broadband sparse phased arrays.

\section{Array Configuration}

A common way to describe a two dimensional spiral is by using polar coordinates $(r, \psi)$, where $r$ is a continuous monotonic function of the angle $\psi$. There are many types of spirals that can be constructed such as Archimedean, logarithmic Euler (wikipedia 2009). A special case of a logarithmic spiral is the Golden Ratio Spiral where its growth factor is related to the Golden Ratio. The polar equation for a Golden Ratio spiral:

$$
\begin{aligned}
& r_{n}=d f(n) \\
& \psi_{n}=2 \pi \phi n
\end{aligned}
$$

where, $r_{n}$ is the radial displacement of the $n^{\text {th }}$ element, $d$ is a scaling factor, $\psi$ is the angle displacement of the $n^{\text {th }}$ element and $\phi$ is the golden ratio $=1.618 \ldots$ (mathworld 2008). Provided 


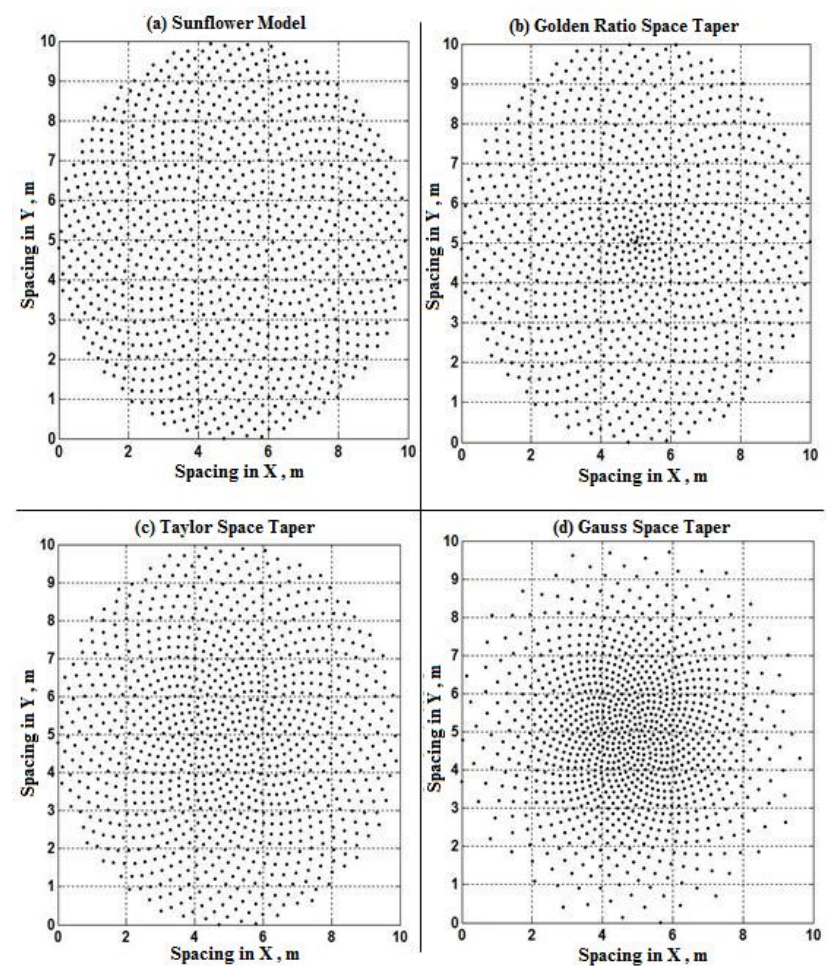

Fig. 1: Array Configurations with Different tapering

that $\phi$ is maintained as the golden ratio, equation (1)\&(2) define a class of array geometry termed here "Golden Ratio Spiral" defined for various functions $f(n)$.

The function $f(n)$ controls the radius of the $n^{\text {th }}$ element from the centre and can be described as a space taper function. It is the design of this function that governs the broadband behaviour of the Golden Ratio spiral class of phased arrays.

The function $f(n)$ can be an analytical function such as the sunflower model in Boeringer (2002).In this configuration $f(n)=\sqrt{n / \pi}$, where $n$ is the index number of the $n^{\text {th }}$ element. Another analytical space taper function found to be useful is $f(n)=n^{(1 / \phi)}$ where, $n$ is the index number of the $n^{\text {th }}$ element,and $\phi$ is the golden ratio. The resultant array is called Golden Ratio Space Taper ${ }^{\mathrm{b}}$.

The function $f(n)$ can also be designed according to a density taper function as in Vigan (2000). A Taylor and a Gauss space tapers are selected to be compared with the golden ratio space taper and the sunflower model described above.

The chosen number of elements is 1020 enclosed in a fixed aperture diameter of $10 \mathrm{~m}$. A regular array with the same number of elements and aperture size has a maximum possible gain at a frequency close to $1 \mathrm{GHz}$. Therefore, a wide bandwidth centred at around $1 \mathrm{GHz}$ is chosen to asses the performances of the different configurations.

The resultant array configurations for different tapers are shown in figure 1 . The figure shows how the space tapering

\footnotetext{
b The Golden Ratio Space Taper array is also a special case of the class of Golden Ratio spiral arrays
}

effects the antenna positions via the geometry equations (1) \& (2).

\section{Array Gain}

The resultant gain for each configuration is plotted as function of frequency in figure 2. In the case of the sunflower model, a high maximum gain is achieved at $f_{\max }=0.95 \mathrm{GHz}$. However, a dramatic drop in gain occurs at higher frequencies. This configuration is useful when a high gain is needed at the low end of the band. The effect of space tapering in the case of the golden ratio and Taylor space taper reduces the maximum gain but provides smoother gain over a wider bandwidth. In the case of a Gauss taper more gain has been further compromised for wider bandwidth. The gain in all configurations tends towards $N x G$ at higher frequencies.

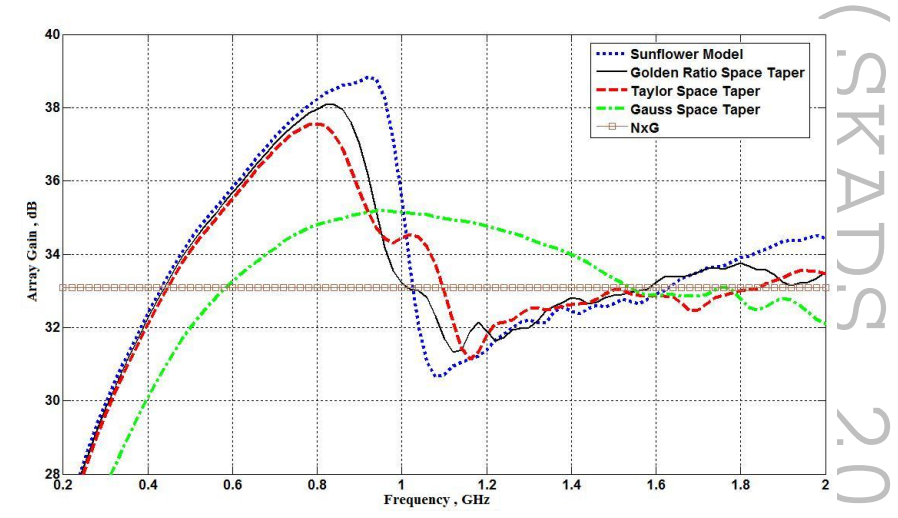

Fig. 2: Gain performance for different space tapering

The average gain for all configurations throughout the bandwidth is the approximately the same. However, it is the behaviour and stability of gain over the bandwidth that can be of interest.

\section{Radiation Patterns}

The radiation patterns for the different configurations are shown in figure 3 at $1 \mathrm{GHz}$ normalized to the peak of the main beam. The side lobe area can be divided into three regions in the form of rings:

1. An inner ring that contains the main beam also contains the near in side lobes (this includes the first side lobe next to main beam). Space tapering such as in a Gauss taper produces a broader main beam with much lower first side lobe of $-39 \mathrm{~dB}$ compared to $-23 \mathrm{~dB}$ in a golden ratio taper configuration. 2 . An outer region of higher sidelobes is formed with different widths and amplitudes for each configuration. High amplitude with a thin width outer ring appears with the sunflower tapered compared to a wider lower amplitude outer ring in the case of Gauss taper. 3. A middle region of lower side lobes remain between regions (1) \& (2), also varying in width and amplitude for the different arrays.

At higher frequencies as shown in figure 4 the outer rings with higher sidelobes widens reducing the size of the middle 

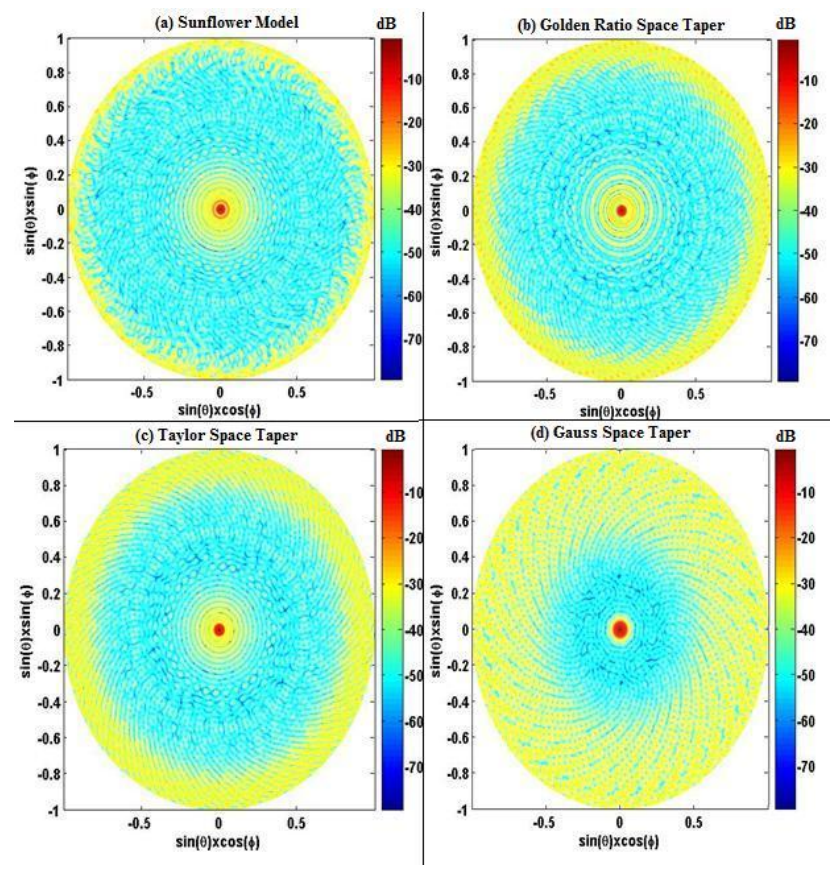

Fig. 3: Radiation Patterns for different configurations at $1 \mathrm{GHz}$

rings of lower sidelobes leaving the level of inner side lobes unaffected despite the narrower main beam. In practice, the results
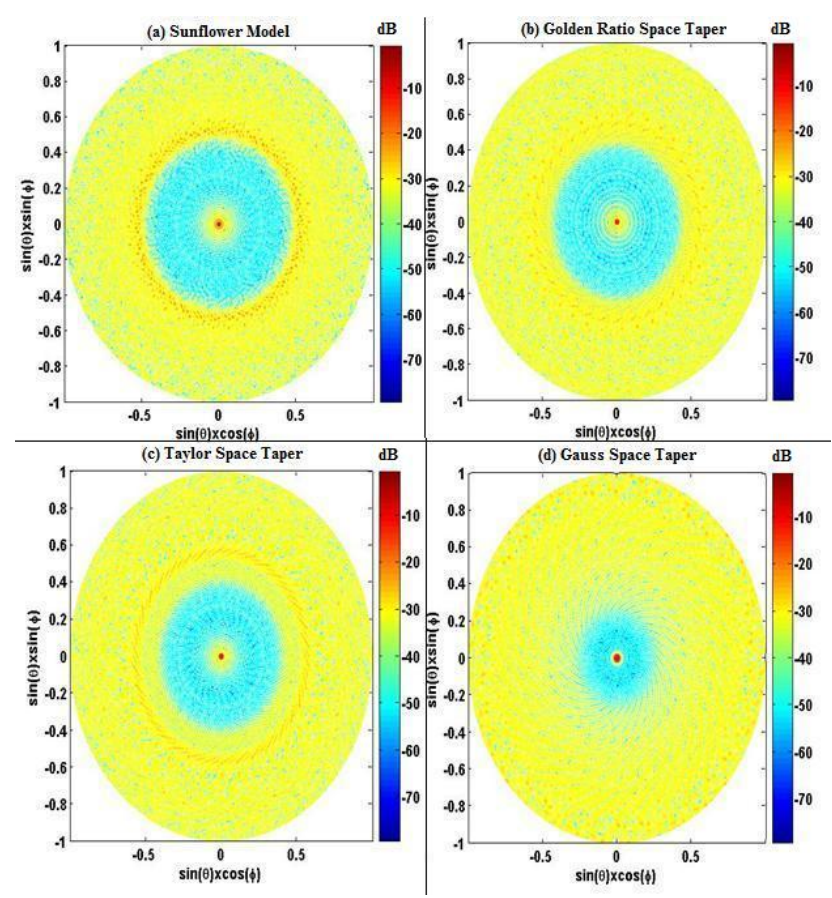

Fig. 4: Radiation Patterns for different configurations at $2 \mathrm{GHz}$

presented in figures 4 and 5 will be multiplied by a realistic element pattern which can in principle be optimized to reduce side lobes at wide angles for a given configuration. However, a narrow element radiation pattern will in general require a larger element which has to comply with the constraints of the array geometry, thus needs a consideration study on a case by cases basis.

Similar to the gain performance, the mean side lobe remains similar irrespective of the array configurations. Once again, it is the location and relative amplitude of side lobe regions that helps to optimizes different designs. The mean side lobe is plotted in figure 6 over the band for different configurations. This remains only marginally affected with the exception of the Gauss taper where at low frequencies, has a broad beam which dominates the radiation pattern.

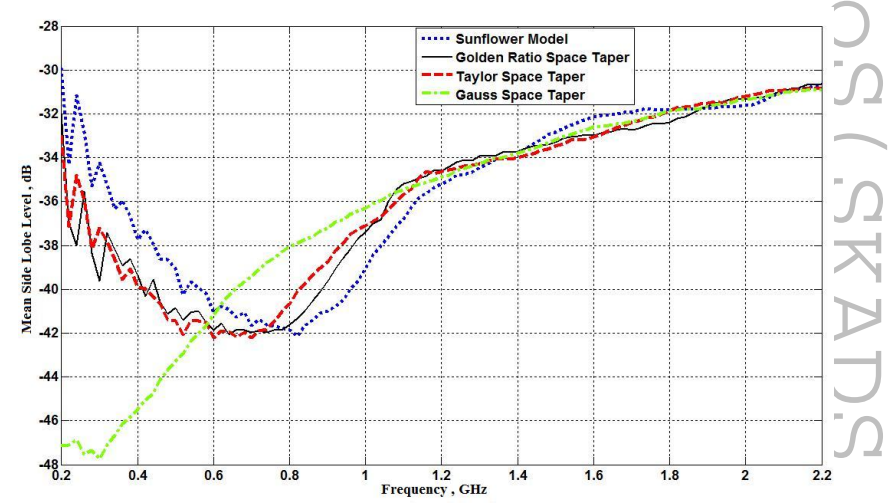

Fig. 5: Mean side lobe performance over a broadband for different configurations

\section{Design Case}

The Square Kilometre Array radio telescope low frequency array is required to operate over a wide band between $700-$ $450 \mathrm{MHz}$. The sky noise at low frequencies is well known to be much higher than upper frequencies (Medellín 2004). The array is required to achieve a minimum of $45 \mathrm{dBi}$ gain at the low frequency to over-come the sky noise. Figure 7 shows the gain performance over the band using the Golden Ratio Space Taper and the Gauss space taper configurations both designed to achieve $45 \mathrm{~dB}$ at $700 \mathrm{MHz}$ using the minimum number of elements. The Golden Ratio Space Taper achieves the required gain using only 4000 elements, whereas the Gauss space taper needs twice the number of elements to meet the required gain. However, the Gauss taper has better gain performance at higher frequency although this gain might not be needed as the sky noise is lower at those frequencies.

\section{Conclusion}

Space taper antenna arrays based on golden ratio configuration exhibit many attractive properties for designing and optimizing broadband arrays for various applications. Different array geometry can be used to trade off the maximum achievable gain at a narrowband for average bandwidth and side lobes. High gain over wider band can be achieved while maintaining good 


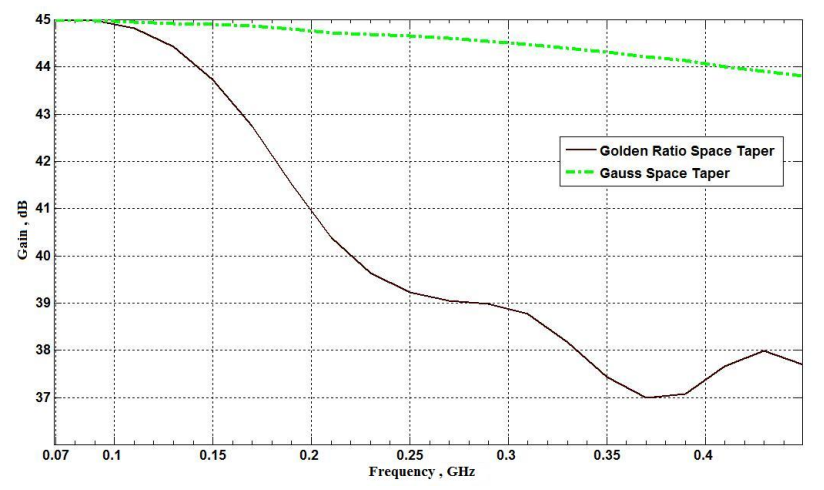

Fig. 6: Gain performances for SKA low frequency array using Golden Ratio and Gauss space taper configurations

control of side lobe amplitude and locations in the radiation pattern.

Acknowledgements. The authors greatly acknowledge the funding and support of the Science and Technology Facilities Council (STFC) in undertaking this work as part of the UK Square Kilometre Array Design Study (SKADS) project.

\section{References}

Boeringer, D.W. "Phased array including a logarithmic spiral lattice of uniformly spaced radiating and receiving elements," U.S. Patent No. 6433745 B1, Silver Spring, MD, US, 13 Apr. 2002.

Chow, Y., "On Grating Plateaux of Nonuniformly Spaced Arrays" IEEE Transactions on Antennas and Propagation, Vol. 13, Is. 2, Mar. 1965 pp: 208 - 215

El-makadema, A., A.K Brown, "Cost Performance Optimization of a Large Scale Wideband Arrays for radio telescope applications," 2008 Loughborough Antenna Propagation Conference p201-p204

Haupt, R.L., "Optimized Element Spacing for Low Sidelobe Concentric Ring Arrays", IEEE Transactions on Antennas and Propagation, Vol. 56, Is. 1, Jan. 2008 pp. 266 - 268.

Howard, W., Sams \& Co., INC, "Reference Data for Radio Engineers", (ITT, Sixth Edition. 1981 ) p 27-25.

Lager, I.E., "The shared aperture, sparse array antennae approach to designing broadband array antennas" Physics and engineering of microwaves, millimetre, and submillimeter waves, the fifth international Kharkov Symposium. Volume 1,2004 issue $21-26$

Mathworld

http://mathworld. wolfram. com/GoldenRatio.html

Cortés Medellín, G., "Antenna Noise Temperature Calculation" National Astronomy and Atmospheric Centre: Cornell University 2004

Vigan, M.C., "Sunflower Array Antenna with Adjustable Density Taper" International Journal of Antennas and Propagation, Volume 2009, Article ID 624035, 10 pages

Wikipedia http://en.wikipedia.org/wiki/Spiral 